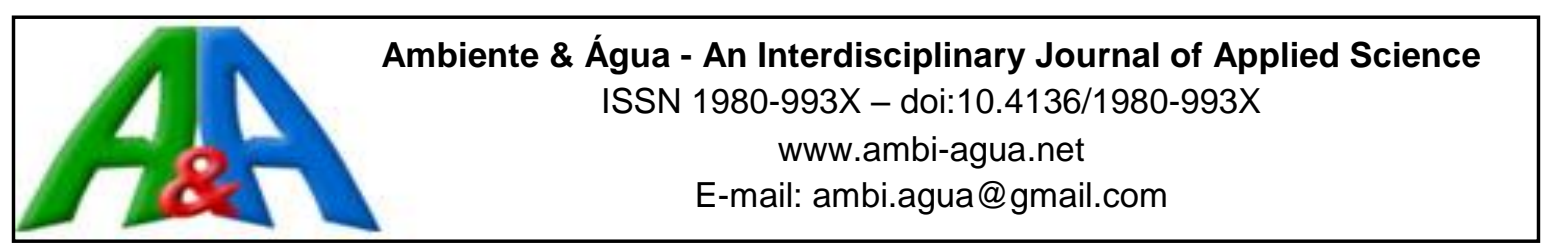

\title{
Vulnerabilidade e mudanças climáticas: análise socioambiental em uma mesorregião da Amazônia
}

\author{
doi:10.4136/ambi-agua.2017
}

Received: 30 Sep. 2016; Accepted: 13 Jun. 2017

\author{
Marcos Ronielly da Silva Santos*; Maria Isabel Vitorino; \\ Marcia Aparecida da Silva Pimentel \\ Universidade Federal do Pará (UFPA), Belém, PA, Brasil \\ Departamento de Geociências \\ *Autor correspondente: e-mail: mronielly@yahoo.com.br, \\ vitorino@ufpa.br, mapimentel@ufpa.br
}

\section{RESUMO}

O estudo da vulnerabilidade socioambiental associado às mudanças climáticas é uma das temáticas mais discutidas pela comunidade científica internacional. Os efeitos das mudanças do clima sobre a sociedade têm origem em diferentes causas, e sua análise deve levar em consideração os contextos cultural, econômico e social de uma população. Deste modo, os estudos envolvendo riscos associados a eventos extremos de clima devem ser feitos de forma integrada e interdisciplinar. Neste contexto, a presente pesquisa visa contribuir para análise da vulnerabilidade na mesorregião metropolitana de Belém, com vistas à redução dos efeitos causados pelas mudanças climáticas e sociais. Para tanto, se aplicou o Índice de Vulnerabilidade Geral (IVG), com base em indicadores socioeconômicos, epidemiológicos e climáticos no período de 2000 a 2010. Os resultados mostram que o município de Belém apresentou o maior valor de IVG $(0,61)$, com alta vulnerabilidade, apresentando os maiores índices de vulnerabilidade climática $(1,00)$ e epidemiológica $(0,76)$. Em contrapartida o município de Bujaru apresentou uma baixa vulnerabilidade, com o valor do IVG $(0,14)$, explicada pelo valor mínimo do índice de vulnerabilidade climática $(0,00)$ e baixos índices de vulnerabilidade epidemiológica $(0,03)$ e socioeconômica $(0,38)$. Espera-se que este estudo possa subsidiar a gestão de políticas públicas para os municípios, e motivar estudos da vulnerabilidade socioambiental na região amazônica, com abordagem interdisciplinar.

Palavras-chave: clima, Belém, risco.

\section{Vulnerability and climate change: socio-environmental analysis in a mesoregion of Amazonia}

\section{ABSTRACT}

The study of socio-environmental vulnerability associated with climate change is one of the topics most discussed by the international scientific community. The effects of climate change on society stem from different causes and effects; their analysis must take into account the cultural, economic and social context of a population. Thus, studies involving risks associated with extreme weather events must be done in an integrated and interdisciplinary way. In this context, the present research aims to contribute to vulnerability analysis in the metropolitan mesoregion of Belém, with a view to reducing the effects caused by climate and social changes. In order to do so, the General Vulnerability Index (GVI) was applied, based on 
socioeconomic, epidemiological and climatic indicators from 2000 to 2010 . The results found that the municipality of Belém had the highest value of GVI (0.61), indicating a high vulnerability, presenting the highest indexes of climatic (1.00) and epidemiological (0.76) vulnerability. On the other hand, the municipality of Bujaru has a low vulnerability, with a GVI value of 0.14 , explained by the minimum value of the index of climate vulnerability $(0.00)$ and low indices of epidemiological (0.03) and socioeconomic vulnerability (0.38). We expect that this study may support the management of public policies for the municipalities and encourage studies of socio-environmental vulnerability in the Amazon region, with an interdisciplinary approach.

Keywords: Belém, climate, risk.

\section{INTRODUÇÃO}

Estudos científicos em vulnerabilidade e adaptação às mudanças climáticas vêm se expandindo nas últimas décadas, devido a grande ocorrência de eventos extremos que afetam diretamente a sociedade humana. Logo, torna-se necessário o conhecimento aprofundado dos possíveis moduladores das vulnerabilidades socioambientais das regiões (Brasil, 2005; Confalonieri et al., 2009; Tibúrcio e Corrêa, 2012). A abordagem do tema da vulnerabilidade no âmbito deste estudo foi feita por meio do conceito clássico de risco, entendido como a probabilidade da ocorrência de um efeito adverso ao bem estar social (Wisner, 2009).

Blaikie et al. (1994) e O'Brien et al. (2013) afirmam que a vulnerabilidade está diretamente associada à capacidade de um grupo em resistir a efeitos nocivos e a perigos, com a possibilidade de se recuperar facilmente. Assim, a vulnerabilidade envolve uma combinação de fatores que determinam o grau, em que um grupo é colocado em risco por um evento discreto e identificável na natureza e/ou sociedade. O conceito de vulnerabilidade socioambiental ascendeu-se amplamente a partir dos anos 80, por diversos teóricos que estabeleceram uma relação de causa e efeito, gerada entre a natureza e a sociedade (D’Ercole, 1994; Maior e Cândido, 2014).

Nesta perspectiva, as mudanças climáticas ganham grande visibilidade, pois trazem profundas implicações sociais, econômicas, políticas e ambientais; interferindo diretamente no grau e/ou percepção de vulnerabilidade socioambiental. Segundo o Painel Intergovernamental de Mudanças Climáticas (IPCC, 2012), as alterações no clima global irão influenciar diretamente na vida das populações dada por diferentes impactos. Neste segmento o Brasil publicou o Plano Nacional sobre Mudança do Clima - PNMC, almejando pôr em prática ações para mitigar os impactos negativos das mudanças climáticas sobre as populações, a fim de criar condições internas para o enfrentamento de suas consequências.

A Amazônia é uma região brasileira que sofre e interfere diretamente nas mudanças do clima, contribuindo diretamente para a emissão de vapor de água para a atmosfera e consequentemente na umidade atmosférica, precipitação e escoamento superficial (Marengo, 2006). O clima da Amazônia é modulado pela interação de diversos fenômenos oceano-atmosfera, e por sistemas atmosféricos que atuam em diferentes escalas espaço-temporais. A precipitação é a variável climatológica mais estudada na região, pois se relaciona com outras características físicas, como a temperatura e umidade (Moraes, 2005; Albuquerque et al., 2010).

A mesorregião metropolitana de Belém se localiza na Amazônia oriental e possui aproximadamente 3 milhões de habitantes, neste subespaço têm sido registrados nos últimos 20 anos, as mais altas taxas de ocupação humana na Amazônia (Castro, 2003; IBGE 2010).

Belém é a capital do estado do Pará, e apresenta um acumulado médio anual de $3001 \mathrm{~mm}$ de chuva, com uma variação de 300 a $450 \mathrm{~mm}$ durante os meses do período chuvoso, se caracterizando como uma das regiões mais chuvosas da Amazônia (Sodré e Rodrigues, 2013). 
Segundo Tavares e Mota (2012) em Belém foram registrados 50 eventos extremos de precipitação, somente no período chuvoso em 20 anos, o aumento no número de eventos extremos de precipitação em uma região é uma das consequências das mudanças no clima.

Neste contexto, é importante o desenvolvimento de estudos que correlacionem metodologias visando mensurar a vulnerabilidade socioambiental, atrelada a componente climática (alto índice de precipitação) e a social (alta densidade demográfica), tendo ambas caráter interdisciplinar (Iwama et al., 2016). A exemplo, vários autores como Deschamps et al. (2009), Alves (2009) e Almeida (2010) desenvolveram pesquisas e metodologias com uso de índices e indicadores para tentar medir o grau de vulnerabilidade socioambientais nos centros urbanos, considerando essas áreas como fontes de pesquisa para estes estudos, pois provocam a concentração de grandes contingentes populacionais, econômicos e ambientais em pequenos espaços urbanos.

O presente trabalho busca mensurar por meio do Índice de Vulnerabilidade Geral (IVG) a vulnerabilidade em uma mesorregião amazônica, com base em indicadores socioeconômicos, epidemiológicos e climáticos, levando em consideração a importância da mesorregião metropolitana de Belém por deter $34 \%$ da população do estado do Pará em apenas $0,6 \%$ do território estadual. Considerando que a ocorrência de eventos extremos de precipitação em centros urbanos, pode elevar os índices de vulnerabilidades em suas múltiplas facetas, deixando a sociedade mais exposta aos riscos socioeconômicos, epidemiológicos e climáticos, provocando mudança na relação sociedade-natureza e nos indicadores socioambientais.

\section{MATERIAIS E MÉTODOS}

\subsection{Caracterização da área de estudo}

A pesquisa abrange a mesorregião metropolitana de Belém, localizada no Nordeste do Estado do Pará, nas coordenadas 0`39'49,72'’S e 47¹7’03,41'’W (Figura 1).

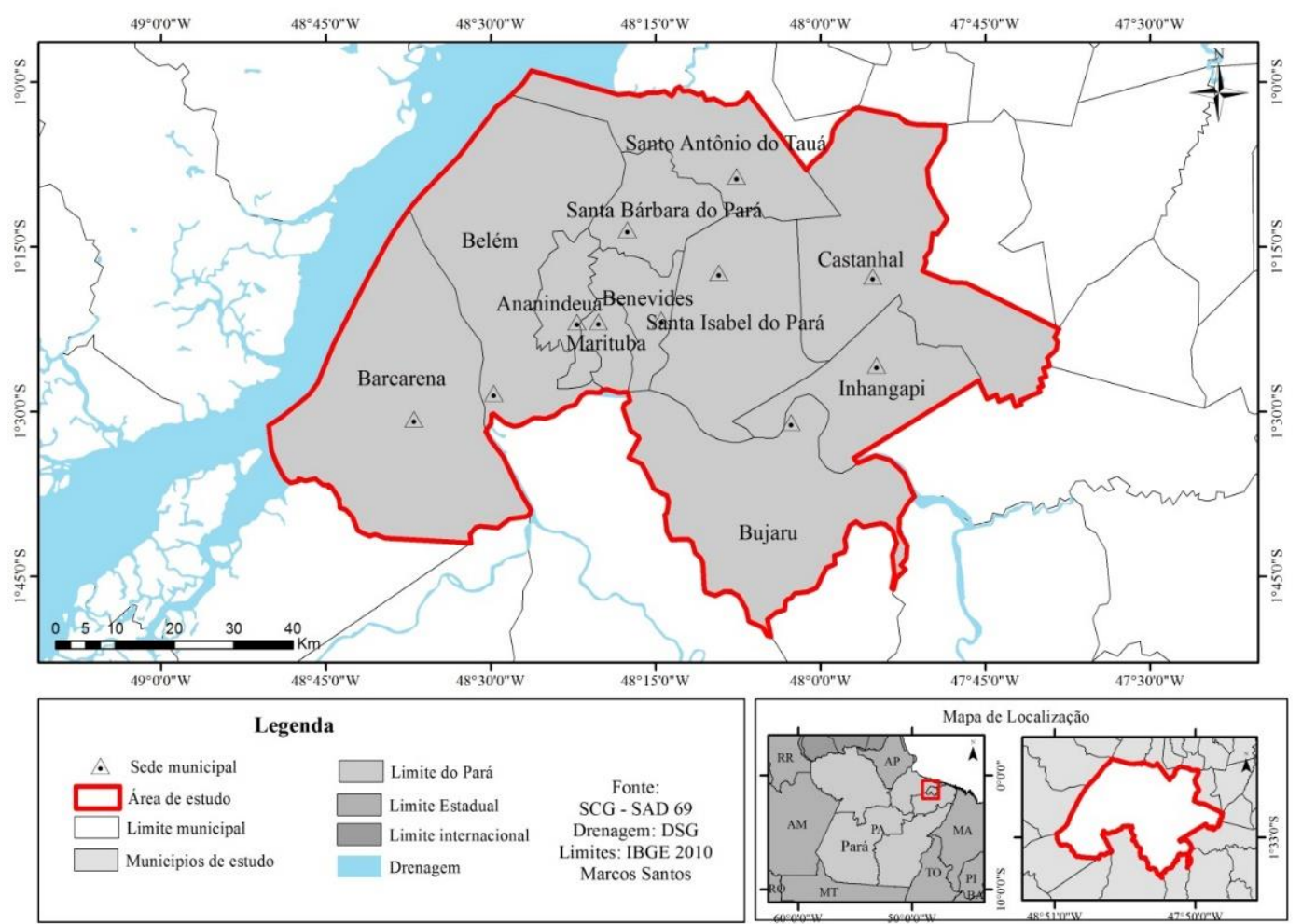

Figura 1. Mapa de localização da área de estudo, com destaque para a mesorregião metropolitana de Belém. 
A mesorregião é formada por onze (11) municípios: Ananindeua, Barcarena, Belém, Benevides, Bujaru, Castanhal, Inhangapi, Marituba, Santa Barbará do Pará, Santa Isabel do Pará e Santo Antônio do Tauá. Estes municípios compreendem uma área territorial de 6.890,336 $\mathrm{km}^{2}$, com população estimada em 2.554.874 habitantes e densidade demográfica de 370,7 $\mathrm{hab} / \mathrm{km}^{2}$ (IBGE, 2010).

A região de estudo possui a classificação climática Afi (tropical úmido), segundo classificação de Köppen é caracterizado por chuvas abundantes durante todo o ano, com período mais chuvoso entre dezembro e maio, e menos chuvoso de junho a novembro, com médias climáticas anuais de $26^{\circ} \mathrm{C}$ de temperatura, $85 \%$ de umidade relativa do ar e $2870 \mathrm{~mm}$ de precipitação pluviométrica anual. A circulação atmosférica local é comandada pelos alísios de NE, desde o Anticiclone dos Açores, responsável pela formação da Massa Equatorial Atlântica (mEa), portadora de abundantes chuvas convectivas (IBGE,1990).

\section{2. Índice de Vulnerabilidade Geral (IVG)}

O IVG foi apresentado no Brasil pelo Ministério de Ciência e Tecnologia e publicado em 2005 em um relatório com o resultado de três anos de pesquisas executadas pela Fundação Oswaldo Cruz (Fiocruz) (Brasil, 2005). Trata-se de uma metodologia aplicada para a avaliação quantitativa da vulnerabilidade por meio da análise de indicadores em três dimensões: socioeconômica, epidemiológica e climática.

O IVG é um índice composto, que agrega diferentes variáveis e associa a cada município da mesorregião de estudo uma medida comparativa (valor numérico) com respeito a sua vulnerabilidade. O IVG é construído pela média aritmética de três outros índices: o Índice de Vulnerabilidade Socioeconômica (IVSE), o Índice de Vulnerabilidade Epidemiológica (IVE) e o Índice de Vulnerabilidade Climática (IVC), de modo que seu valor varia entre 0 e 1 , onde o valor 0 identifica menor vulnerabilidade e o valor 1, maior vulnerabilidade (Brasil, 2005; Confalonieri et al., 2009).

\subsection{1. Índice de Vulnerabilidade Socioeconômica (IVSE)}

O IVSE foi desenvolvido com o objetivo de correlacionar vários indicadores socioeconômicos num indicador sintético. Estes indicadores têm como objetivo concentrar o maior número de informação numa única variável, permitindo comparar elementos, indivíduos ou unidades tanto a nível transversal como temporal. A construção do IVSE consiste em quatros etapas:

Primeira etapa: selecionar os indicadores utilizados e agrupá-los em dimensões. Neste trabalho foram considerados 10 (dez) indicadores distribuídos em 05 (cinco) categorias, cujas definições estão disponíveis no site http://cidades.ibge.gov.br, a saber:

1- Demografia: densidade demográfica $\left(\right.$ hab. $/ \mathrm{km}^{2}$ ) e o grau de urbanização (\%);

2- Renda: população acima de 10 anos com rendimento mensal de $1 / 2$ a 1 salário mínimo (\%) e população acima de 10 anos com rendimento mensal de 2 a 3 salários mínimos (\%);

3- Educação: população acima de 10 anos de idade sem instrução e ensino fundamental incompleto (\%) e população acima de 10 anos de idade com ensino médio completo e ensino superior incompleto $(\%)$;

4- Saneamento: domicílios que possuem e não possuem abastecimento de água (\%), esgotamento sanitário (\%) e coleta de resíduos (\%);

5- Saúde: taxa de mortalidade infantil com menos de 01 (um) ano de idade (\%) e esperança de vida ao nascer $(\%)$. 
Os valores dos indicadores foram retirados do censo demográfico 2010, do Instituto Brasileiro de Geografia e Estatística (IBGE) e do atlas de desenvolvimento humano no Brasil, também referente ao ano de 2010, disponível em http://atlasbrasil.org.br (IBGE, 2010).

Segunda etapa: classificar e padronizar os indicadores segundo sua relação com o conceito de vulnerabilidade apresentado neste estudo, de forma que seus valores variem entre 0 e 1 . Se o valor alto do indicador representa uma situação de menor vulnerabilidade, deve-se utilizar a equação tipo 1. Se o valor baixo do indicador representa uma situação de menor vulnerabilidade, deve-se utilizar a equação tipo 2. Nesta etapa tende-se a desconsiderar as dimensões dos indicadores, tornando-os adimensionais, a partir das seguintes Equações 1 e 2.

Tipo 1: Ip $=\frac{\text { áximo }_{I}-I_{\text {observado }}}{\text { Máximo }_{I-\text { Minimo }_{I}}}$

Tipo 2: Ip $=\frac{I_{\text {observado }}-\text { Mínimo }_{I}}{\text { Máximo }_{I-\text { Minimo }}}$

em que:

Ip é cada um dos 10 indicadores analisados; Iobservado é o valor observado do indicador I;

Mínimo é o menor valor observado do indicador I; e Máximo é o maior valor observado I.

Terceira etapa: construir um índice sintético por dimensão (demografia, renda, educação, saneamento e renda), dado pela média aritmética simples dos indicadores analisados.

Quarta etapa: desenvolver o IVSE por meio da média aritmética simples dos resultados das dimensões.

\subsection{2. Índice de Vulnerabilidade Epidemiológica (IVE)}

O IVE foi construído com o propósito de sintetizar, as informações contidas num grupo de três doenças de veiculação hídrica em um único indicador, levando em consideração a relação entre clima - inserindo eventos extremos de chuva e surtos de endemias. As doenças selecionadas são: Dengue, Leptospirose e Hepatite A. A escolha dessas endemias está relacionada à alta incidência de vetores e casos associados a enchentes e inundações na mesorregião de estudo (Travassos da Rosa et al., 2000; Gonçalves et al., 2016).

A construção do IVE constitui-se de três etapas, diferenciando-se dos métodos utilizados por Brasil (2005) e Tibúrcio e Corrêa (2012), pois optou-se não atribuir pesos diferenciados a cada endemia, entendendo que as mesmas devem ser postas de forma iguais na verificação do IVE.

Primeira etapa: Escolha dos indicadores a serem utilizados. Nesta etapa, verificam-se, as informações disponíveis e discute-se a forma de agregá-las à informação individual de cada variável no índice. Nesta pesquisa optou-se por: 1 - Taxa de incidência; 2 - Número de internação/ Número de internação da mesorregião; 3 - Número de óbitos/ Número de óbitos da mesorregião e 4 - Custo de internação/ Custo de internação da mesorregião. Os dados que compões os indicadores foram fornecidos pela Secretaria de Estado de Saúde Pública (SESPA) e pela Diretoria Desenvolvimento de Acompanhamento dos Serviços de Saúde (DDASS) retidos pela fonte SIH/DATASUS, compreendendo os anos de 2000 a 2010.

Segunda etapa: Padronizar os indicadores de acordo com a Equação 3.

$$
\text { Tipo 3:Ip }=\frac{I_{\text {observado }}-\text { Mínimo }_{I}}{\text { Maxímo }_{I}-\text { Mínimo }_{I}}
$$


Terceira etapa: Construir o índice sintético de cada endemia por meio da média aritmética simples dos indicadores padronizados.

\subsection{3. Índice de Vulnerabilidade Climática (IVC)}

O IVC é apresentado com o objetivo de classificar os municípios conforme o número (\%) de meses que apresentam os níveis extremos de precipitação. Em outras palavras, os eventos extremos representam uma maior exposição da sociedade á vulnerabilidade socioeconômica e epidemiológica. A construção do IVC consiste em duas etapas:

Primeira etapa: reconhecer os valores "extremos". Neste trabalho, a avaliação de valores extremos adotou os seguintes critérios: "extremo alto" é um valor observado quando a precipitação for maior que a soma do terceiro quartil mais 1,5 vezes a distância interquartil da amostra considerada. Por outro lado, "extremo baixo" é um valor observado quando a precipitação for menor que a soma do primeiro quartil menos 1,5 vezes a distância interquartil da amostra (Magalhães e Lima, 2002). Os valores foram observados a partir da construção de boxplot (diagramas de caixa), uma ferramenta comum e simples para a identificação de valores extremos, que considera a assimetria e a variabilidade de um determinado conjunto de dados (Triola, 2008).

Segunda etapa: consideração da porcentagem de meses que apresentam acumulados de precipitação extremos, calculado a partir da seguinte Equação 4.

$$
\text { Tipo 4: } I V C=\frac{P_{\text {observada }}-\text { Mínimo }}{\text { Máximo-Mínimo }}
$$

em que:

Pobservada refere-se ao valor da porcentagem de meses com precipitação extrema alta;

Mínimo refere-se ao menor valor observado de meses com precipitação extrema, e

Máximo refere-se ao maior valor observado de meses com precipitação extrema.

Os dados utilizados para a construção dos boxplot foram retirados das séries mensais de precipitação, dados pela técnica CPC MORPHing technique (CMORPH), que consiste em uma estimativa de precipitação, dada pela leitura de microondas passivas por satélite geoestacionário. Esses dados são estimados e especializados sob áreas que não possuem estações e/ou pluviômetros de medição superficial (Joyce et al., 2004). Os dados climáticos compreendem uma resolução espacial de 8 por 8 quilômetros em pontos de grade sobre a área de estudo e uma resolução temporal de cada 30 minutos durante o período de janeiro de 2000 a dezembro de 2010.

\subsubsection{Análise estatística}

Os municípios foram agrupados em três grupos: alta, média e baixa vulnerabilidade segundo suas homogeneidades, utilizando-se a técnica estatística de análise hierárquica de cluster, a qual consiste em um processo de partição de população heterogênea em vários subgrupos mais homogêneos, buscando agrupar elementos de dados baseando-se na similaridade entre eles (Zaiane e Oliveira, 2003). A medida de similaridade entre dois elementos adotada foi a distância euclidiana, utilizando o método da ligação entre grupos ou ligação por média, que trata o espaço entre dois conglomerados como a média das distâncias entre todos os pares de elementos que podem ser formados com os elementos comparados. (Mingoti, 2005). 


\section{RESULTADOS E DISCUSSÃO}

A Tabela 1 mostra os valores dos índices sintéticos de cada dimensão analisada (socioeconômica, epidemiológica e climática), bem como os valores padronizados de cada indicador ou variável que compuseram os referidos índices.

Tabela 1. Índices de vulnerabilidade por municípios e indicadores padronizados.

\begin{tabular}{lccccccccccccc}
\hline Município & Demografia & Renda & Educação & Saneamento & Renda & IVSE & Dengue Leptospirose & Hepatite & IVE & IVC & IVG \\
\hline Ananindeua & 0,00 & 0,04 & 0,06 & 0,31 & 0,06 & 0,09 & 0,59 & 0,18 & 0,19 & 0,31 & 0,71 & 0,37 \\
Barcarena & 0,45 & 0,10 & 0,50 & 0,49 & 0,26 & 0,35 & 0,20 & 0,06 & 0,08 & 0,11 & 0,57 & 0,34 \\
Belém & 0,27 & 0,14 & 0,00 & 0,00 & 0,00 & 0,08 & 0,48 & 0,97 & 0,84 & 0,76 & 1,00 & 0,61 \\
Benevides & 0,35 & 0,35 & 0,48 & 0,48 & 0,29 & 0,39 & 0,25 & 0,05 & 0,10 & 0,13 & 0,14 & 0,22 \\
Bujaru & 0,47 & 0,01 & 0,46 & 0,45 & 0,55 & 0,38 & 0,08 & 0,02 & 0,01 & 0,03 & 0,00 & 0,14 \\
Castanhal & 0,11 & 0,35 & 0,48 & 0,43 & 0,20 & 0,31 & 0,06 & 0,04 & 0,14 & 0,08 & 0,42 & 0,27 \\
Inhangapi & 0,00 & 0,89 & 0,44 & 0,80 & 0,00 & 0,42 & 0,02 & 0,00 & 0,08 & 0,03 & 0,14 & 0,20 \\
Marituba & 0,21 & 0,22 & 0,32 & 0,51 & 0,36 & 0,32 & 0,24 & 0,20 & 0,13 & 0,19 & 0,00 & 0,17 \\
Sta Barbará & 0,48 & 0,35 & 0,68 & 0,19 & 0,48 & 0,43 & 0,07 & 0,00 & 0,10 & 0,05 & 0,57 & 0,35 \\
Sta Isabel & 0,20 & 0,41 & 0,63 & 0,60 & 0,29 & 0,42 & 0,30 & 0,05 & 0,10 & 0,15 & 0,57 & 0,38 \\
Sto Antônio & 0,31 & 0,36 & 0,70 & 0,58 & 0,92 & 0,57 & 0,10 & 0,01 & 0,07 & 0,06 & 0,42 & 0,35 \\
\hline
\end{tabular}

A priori, no intuito de melhor compreender os resultados da análise, a mesma será feita de forma integrada por meio do mapa de vulnerabilidade geral e posteriormente de forma separada em cada uma das dimensões analisadas, a fim de, especificar qual índice ou indicador elevou a vulnerabilidade na categoria pesquisada.

A Figura 2 apresenta o mapa de vulnerabilidade geral. Neste, o município de Belém mostrou o maior valor de IVG $(0,61)$ (Tabela 1$)$, designando uma alta vulnerabilidade, carreado pelos altos índices de vulnerabilidade climática $(1,00)$ e epidemiológica $(0,76)$. Segundo Mendonça (2005), acredita-se que as correlações dos fatores climáticos e epidemiológicos elevam a vulnerabilidade de populações humanas em face de condições extremas. Neste caso, o alto IVC apresentado por Belém está associado com a grande quantidade de chuva precipitante no município, oriundo da interação de escalas e sistemas precipitantes locais (Matos e Cohen, 2016). O alto IVE deve-se ao grande número de casos das endemias. Os registros dessas endemias bem como os outros fatores que compõem o índice apresentaram valores máximos para o município de Belém, devido ao fato deste ser a capital do estado do Pará e apresentar-se como município de referência estadual, promovendo a notificação dos municípios adjacentes.

Observa-se também que há uma diferença significativa entre os valores de IVG de Belém em comparação aos demais municípios, denotando que o IVC e IVE contribuíram significativamente para o aumento da vulnerabilidade geral do município, tornando-se as duas principais dimensões que elevam a vulnerabilidade socioambiental. Em contrapartida o município de Bujaru mostra o menor valor do IVG $(0,14)$, elucidando uma baixa vulnerabilidade nas dimensões analisadas, isto pode ser explicado pelo mínimo valor de IVC $(0,00)$ e baixos índices do IVE $(0,03)$ e IVSE $(0,38)$. 


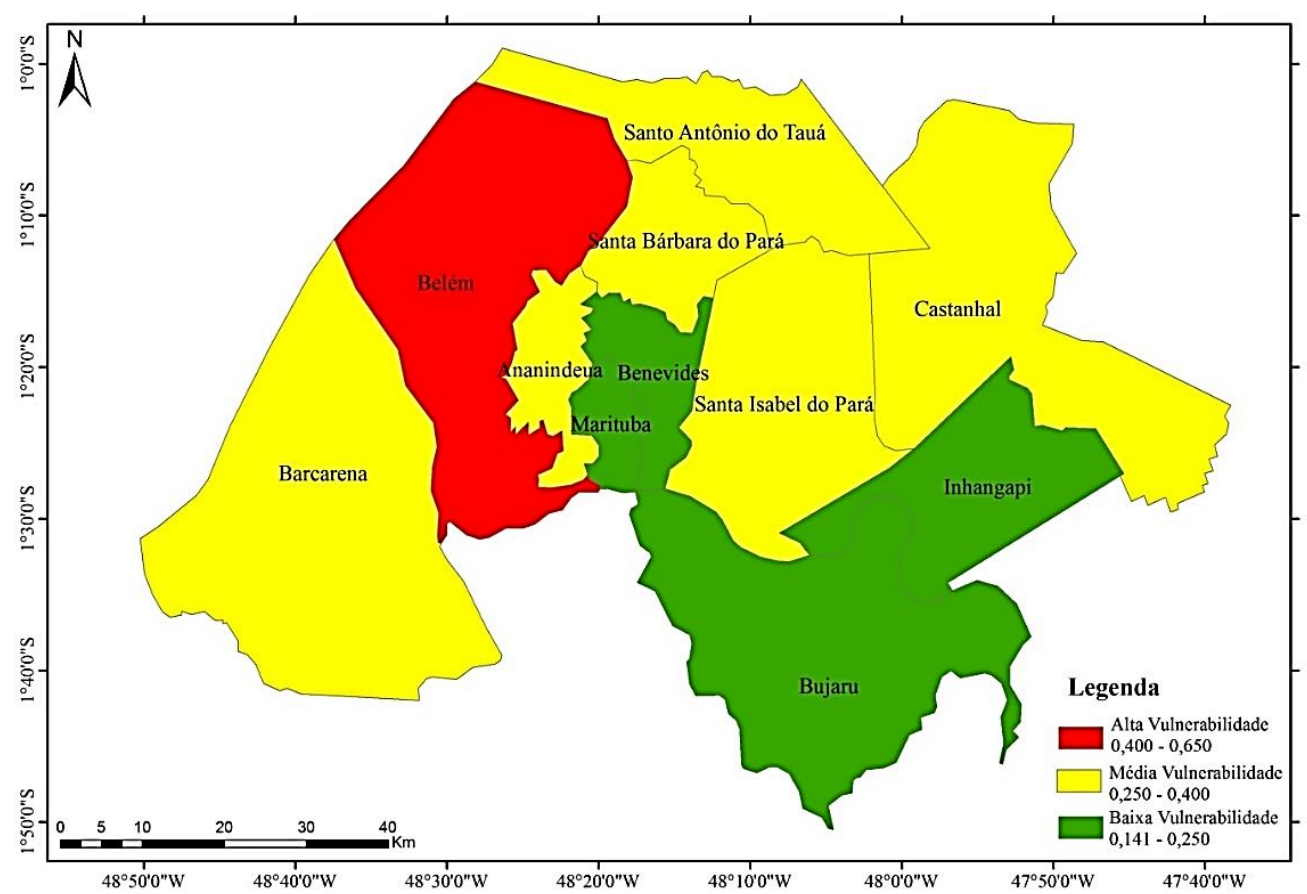

Figura 2. Mapa de vulnerabilidade geral da mesorregião metropolitana de Belém, referente a uma década.

A Figura 3 mostra que o município de Santo Antônio do Tauá foi considerado de alta vulnerabilidade a partir dos indicadores utilizados, pois obteve o mais elevado valor de IVSE da mesorregião $(0,57)$, indicando que em relação aos demais municípios é o que têm a pior situação de vulnerabilidade para esta categoria. O alto índice de Santo Antônio do Tauá pode ser explicado pelos altos valores apresentados nas dimensões de educação, com a segunda maior população acima de 10 anos sem instrução e na dimensão de saúde, com $49 \%$ da população com esperança de vida abaixo dos 70 anos.

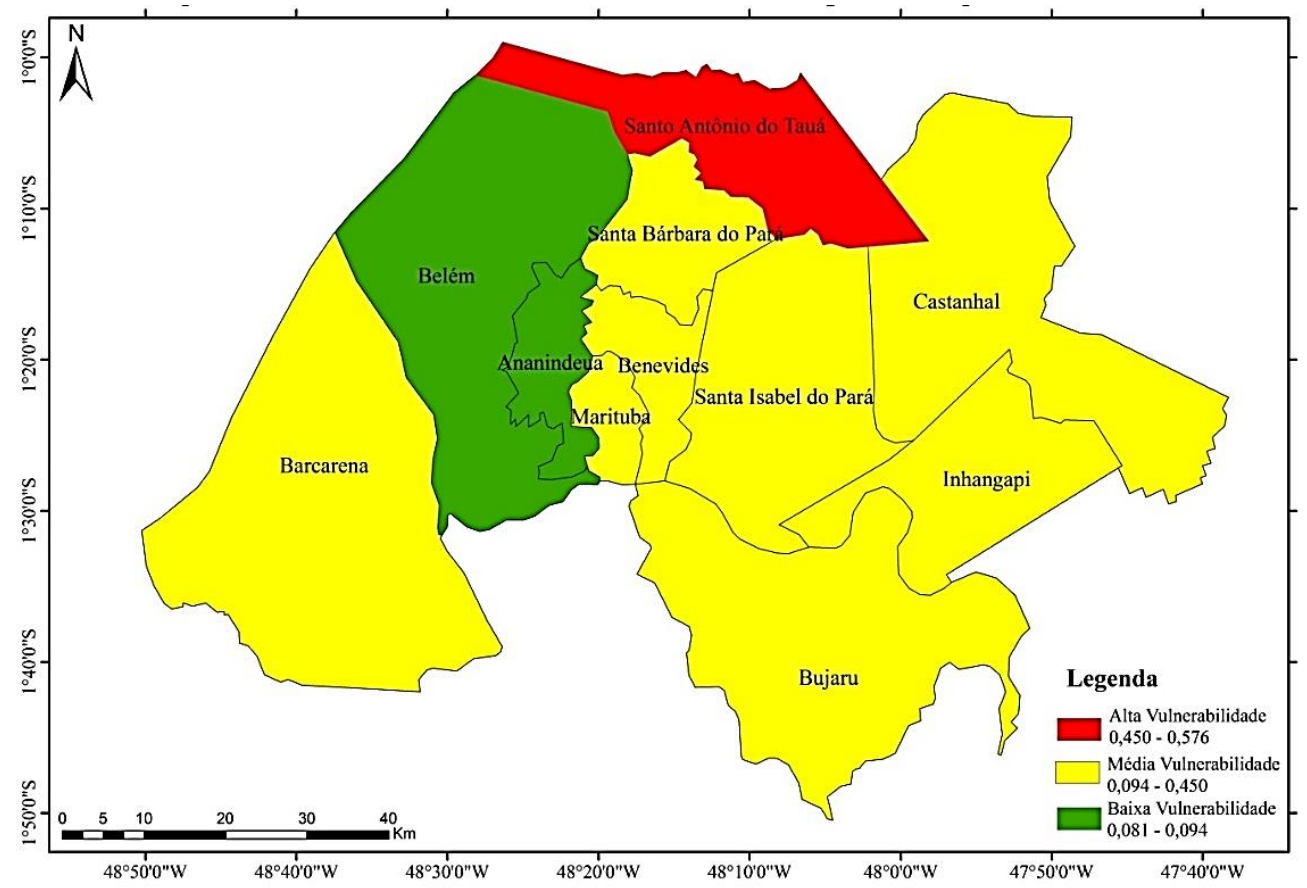

Figura 3. Mapa de vulnerabilidade socioeconômica da mesorregião metropolitana de Belém, referente a uma década. 
Em contrapartida, os municípios de Belém e Ananindeua apresentam os menores índices de vulnerabilidade socioeconômica de 0,08 e 0,09, respectivamente. O baixo IVSE de Belém explica-se, pois a cidade tem um dos maiores graus de urbanização do estado $(99,1 \%)$, a menor taxa da população acima de 10 anos sem instrução e ensino incompleto (32,6\%), e 53,4\% dos domicílios possui abastecimento de água encanada, com esgoto sanitário e coleta de lixo.

Outra variável que se mostrou bastante relevante para o baixo IVSE foi à dimensão da saúde, considerando a menor taxa de mortalidade infantil $(16,1 \%)$ e a maior esperança de vida ao nascer $(74,3 \%)$ dentre os municípios estudados. Conforme corrobora o estudo de Pereira e Vieira (2016), analisando a sustentabilidade da região metropolitana de Belém, os mesmos municípios (Belém e Ananindeua) apresentaram os melhores índices de desenvolvimento humano municipal, quando analisados indicadores semelhantes.

A Figura 4 mostra o mapa de vulnerabilidade epidemiológica. Nele, observa-se que o município de Belém elucida a maior vulnerabilidade epidemiológica $(0,76)$, este valor elevado deve-se ao índice de leptospirose $(0,97)$ e hepatite A $(0,84)$. A leptospirose em Belém registrou $76 \%$ dos óbitos, $90 \%$ das internações e $70 \%$ nos investimentos para internação, se comparada aos demais municípios da mesorregião. O alto índice de hepatite A está associado aos $73 \%$ do número de óbitos, $72 \%$ do número de internação e $40 \%$ nos investimentos em internações registradas durante os onze anos de estudo para o município de Belém.

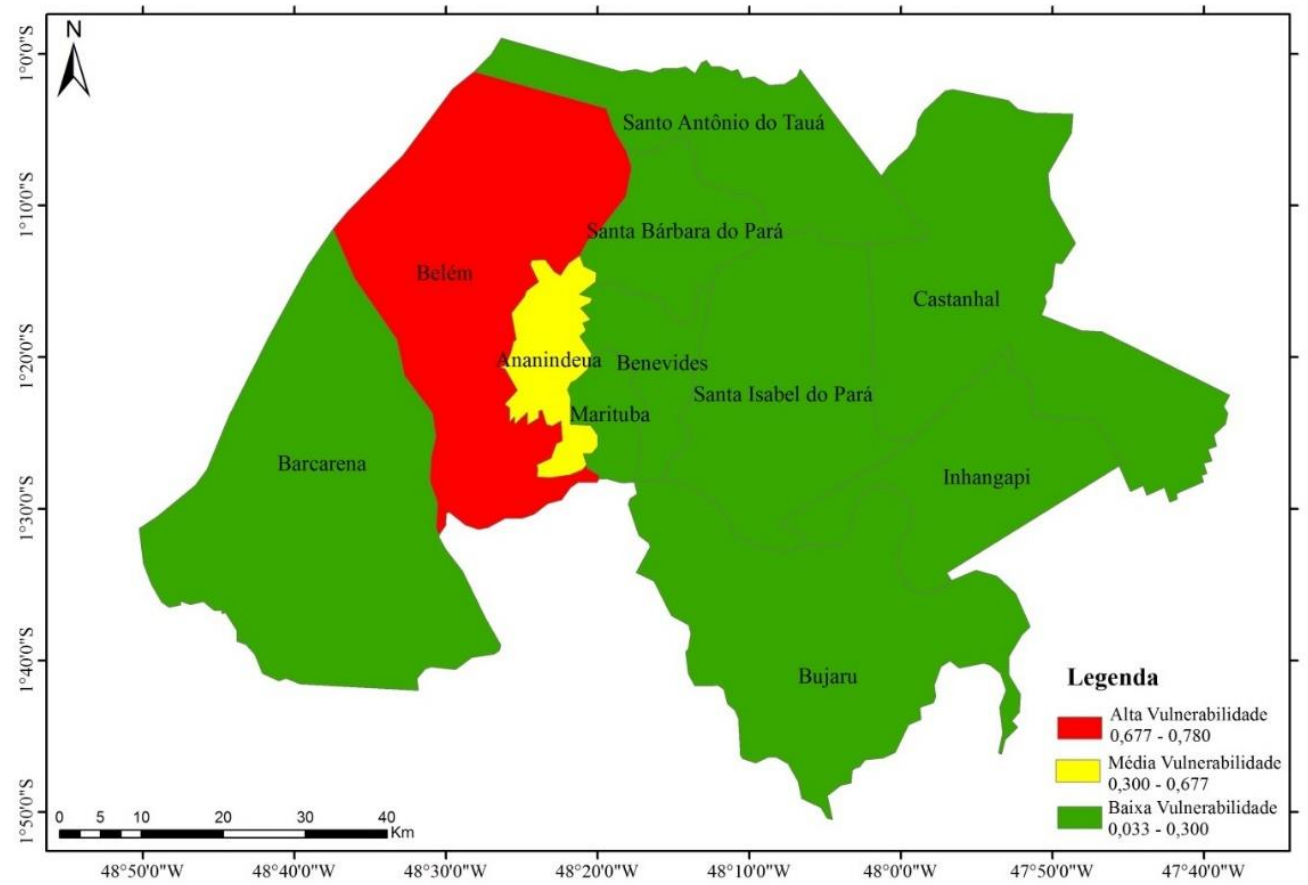

Figura 4. Mapa de vulnerabilidade epidemiológica da mesorregião metropolitana de Belém, referente a uma década.

A alta vulnerabilidade de Belém é resultado da diminuição e/ou à não notificação dos indicadores analisados nos municípios, que apresentaram uma baixa vulnerabilidade epidemiológica. A cidade de Belém se apresenta como centro de referência em saúde, levando a população dos municípios adjacentes a se estabeleceram na cidade. De acordo com Deschamps et al. (2009), a falta de infraestrutura nas cidades pode elevar a vulnerabilidade. Neste caso, a boa infraestrutura hospitalar presente na capital condiciona a notificação de casos dos municípios com pouca infraestrutura.

Em oposição, 9 municípios da mesorregião (Barcarena, Benevides, Bujaru, Castanhal, Inhangapi, Marituba, Santa Barbara do Pará, Santa Isabel do Pará e Santo Antônio do Tauá) 
apresentam uma baixa vulnerabilidade, tendo seu IVE próximo de 0,033 e 0,300. Esse resultado deve-se principalmente aos valores dos índices de dengue, considerando o menor valor para o município de Castanhal $(0,06)$, como consequência da baixa incidência, em torno de $8 \%$ da população. Os baixos valores de leptospirose para os municípios de Santa Bárbara do Pará e Inhangapi $(0,00)$ foram obtidos pela ausência de notificação (Pará, 2016). Os municípios de Bujaru e Santo Antônio do Tauá apresentaram os menores índices de Hepatite A $(0,01$ e 0,07), respectivamente, registrando ambos apenas $1 \%$ das internações durante os anos pesquisados.

A Figura 5 apresenta o mapa da vulnerabilidade climática dos municípios da mesorregião metropolitana de Belém. Nele destaca-se o município de Belém, que apresenta uma alta vulnerabilidade climática $(1,00)$, possuindo 5,30\% das 132 observações. Estudos de Tavares e Mota (2012) mostram que somente a cidade de Belém apresentou mais de 50 eventos extremos de precipitação em apenas 20 anos. O extremo positivo de chuva registrado em Belém, no mês de setembro de 2000, pode estar associado ao efeito do El Niño-Oscilação Sul (ENOS) em sua fase fria (La Niña). Este fenômeno provoca maiores quantidades de precipitação na Amazônia (De Souza et al., 2000). Neste mesmo ano, foi registrado o maior número de casos confirmados de leptospirose no município de Belém (Pará, 2016).

O outro evento extremo negativo, que significa chuva abaixo do padrão sazonal, aconteceu em janeiro (período chuvoso) de 2005, ano da fase quente do ENOS (El Niño). Todavia, segundo Marengo et al. (2008), as causas de seca e a redução de precipitação para a Amazônia no ano de 2005 estão associadas a três possíveis fatores: (1) o Atlântico norte tropical anomalamente mais quente do que o normal, (2) a redução na intensidade do transporte de umidade pelos alísios de nordeste em direção ao sul da Amazônia durante o pico da estação de verão, e (3) a diminuição do movimento vertical sobre esta parte da Amazônia, resultando num reduzido desenvolvimento convectivo e reduzida precipitação.

Noutro sentido, os municípios de Benevides, Bujaru, Inhangapi e Marituba chamaram atenção por apresentarem os menores valores, com percentuais nos meses extremos de precipitação ( $0,75 \%$ das 132 observações) e seu IVC variando em torno de 0,00 e 0,14 (Tabela 1).

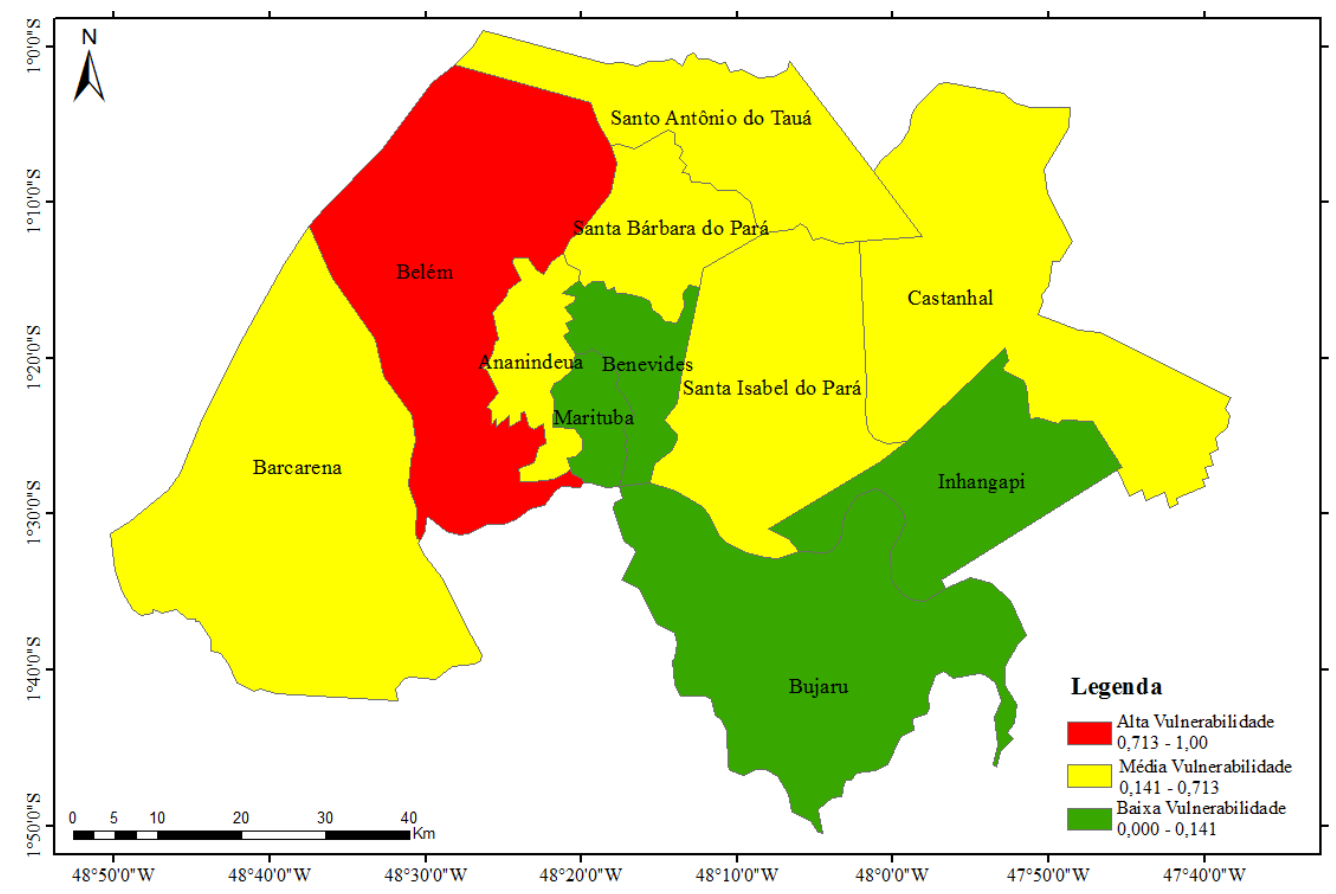

Figura 5. Mapa de vulnerabilidade climática da mesorregião metropolitana de Belém, referente a uma década. 


\section{CONCLUSÃO}

Nota-se que a dimensão mais acentuada para o aumento do Índice de Vulnerabilidade Geral foi a condição climática, apresentando resultado expressivo para 07 dos 11 municípios (Ananindeua, Barcarena, Belém, Castanhal, Santa Barbará do Pará, Santa Isabel do Pará e Santo Antônio do Tauá), tornando-se a principal contribuinte para o aumento da vulnerabilidade geral. As principais conclusões do estudo, considerando os mais baixos e os mais altos índices, são:

- O município de Santo Antônio do Tauá tem uma alta vulnerabilidade socioeconômica, motivada pela baixa escolaridade e esperança de vida. Em contrapartida, os municípios de Belém e Ananindeua têm a uma baixa vulnerabilidade socioeconômica da mesorregião, vista a partir dos indicadores utilizados, conduzida pelo elevado grau de urbanização e alta escolaridade.

- O município de Belém é o mais vulnerável às epidemiologias estudadas, principalmente à leptospirose e Hepatite $\mathrm{A}$, influenciado também pela melhor infraestrutura comparada aos demais municípios. Todos os municípios adjacentes a Belém e Ananindeua tiveram uma baixa vulnerabilidade Epidemiológica, promovidos pelos baixos índices de dengue e nulidade dos dados.

- A vulnerabilidade climática é mais alta no município de Belém, pois apresentou os maiores percentuais de eventos extremos promovidos por moduladores climáticos. Em compensação, a baixa vulnerabilidade nos municípios de Bujaru e Marituba foi promovida pela não ocorrência de eventos extremos de precipitação durante o período analisado.

- A escala temporal dos dados analisados pode interferir nos valores dos índices e, consequentemente, na condição da vulnerabilidade socioeconômica, epidemiológica e climática.

\section{AGRADECIMENTOS}

À Coordenação de aperfeiçoamento pessoal do Ensino Superior (CAPES).

\section{REFERÊNCIAS}

ALBUQUERQUE, M. F.; SOUZA, E. B.; OLIVEIRA, M. do C. F.; SOUZA JÚNIOR, J. A. de. Precipitação nas mesorregiões do estado do Pará: climatologia, variabilidade e tendências nas últimas décadas (1978-2008). Revista Brasileira de Climatologia, ano 6 , n. 6, p. 151-163, 2010. http://dx.doi.org/10.5380/abclima.v6i0.25606

ALMEIDA, L. Q. de. Vulnerabilidade socioambiental de rios urbanos: bacia hidrográfica do Rio Maranguapinho região metropolitana de Fortaleza-Ceará, 2010, 310f. Tese (Doutorado em Geografia) - Universidade Estadual Paulista, Rio Claro, 2010.

ALVES, H. P. F. Metodologias de integração de dados sociodemográficos e ambientais para análise da vulnerabilidade socioambiental em áreas urbanas no contexto das mudanças climáticas. In: HOGAN, D.; MARANDOLA JR, E. (Orgs.). População e mudança climática: dimensões humanas das mudanças ambientais globais. Campinas: Núcleo de Estudos de População - NEPO/Unicamp; Brasília: UNFPA, 2009. p. 75-105.

BLAIKIE, P. M.; CANNON, T.; DAVIS, I.; WISNER, B. Atrisk: natural hazards, people's vulnerability, and disasters. London: Routledge, 1994. 
BRASIL. Análise da vulnerabilidade da população brasileira aos impactos sanitários das mudanças climáticas. Brasília, 2005.

CASTRO, E. Geopolítica da agua e novos dilemas a propósito da Amazônia e seus recursos naturais. In: ARAGON, L. E.; CLÜSENER-GODT, M. (Orgs.) Problemática do uso local e global da água da Amazônia. Belém: NAEA, 2003. p. 334.

CONFALONIERI, U. E. C.; MARINHO, D. P.; RODRIGUEZ, R. E. Public health vulnerability to climate change in Brazil. Climate Research, v. 40, n. 23, p. 175-186, 2009. https://doi.org/10.3354/cr00808

D’ERCOLE, R. Les vulnérabilités des sociétés et des espaces urbanisés: concepts, typologie, modes d'analyse. Revue de Géographie Alpine. v. 82, n. 4, p. 87-96, 1994.

DE SOUZA, E. B.; KAYANO, M. T.; TOTA, J.; PEZZI, L.; FISCH, G.; NOBRE, C. On the influences of the El Niño, La niña and Atlantic Dipole Paterni on the Amazonian Rainfall during 1960-1998. Acta Amazônica, v. 30, n. 2, p. 305-318, 2000. http://dx.doi.org/10.1590/1809-43922000302318

DESCHAMPS, M. V.; DELGADO, P. R.; SUGAMOSTO, M.; DE ULHÔA CINTRA, A. P.; IGNÁCIO, S. A.; RODRIGUES, J. M. et al. Vulnerabilidade socioambiental nas regiões metropolitanas brasileiras. Fortaleza: Observatório das MetrópolesIPPUR/FASE, 2009.

GONÇALVES, N. V.; DE ARAUJO, E. N.; DA SILVA SOUSA, J.; PEREIRA, W. M. M.; DO SOCORRO CARVALHO MIRANDA, C.; DA SILVIA CAMPOS, P. S. et al. Distribuição espaço-temporal da leptospirose e fatores de risco em Belém, Pará, Brasil. Revista Ciência \& Saúde Coletiva, v. 21, n. 12. p. 3947-3955, 2016.

INSTITUTO BRASILEIRO DE GEOGRAFIA E ESTATÍSTICA. Censo demográfico. Rio de Janeiro, 2010.

INSTITUTO BRASILEIRO DE GEOGRAFIA E ESTATÍSTICA. Projeto zoneamento das potencialidades dos recursos naturais da Amazônia Legal. Rio de Janeiro, 1990. 212p.

INTERGOVERNMENTAL PANEL ON CLIMATE CHANGE. Managing the risks of extreme events and disasters to advance climate change adaptation: a special report of working Groups I and II of the IPCC. Cambridge: University Press, 2012. 582 p.

IWAMA, A. Y.; BATISTELLA, M.; DA COSTA FERREIRA, L.; ALVES, D. S.; DA COSTA FERREIRA, L. Risco, vulnerabilidade e adaptação às mudanças climáticas: uma abordagem interdisciplinar. Ambiente \& Sociedade, v. 19, n. 2, p. 95-118, 2016.

JOYCE, R. J.; JANOWIAK. J. E.; ARKIN. P. A.; XIE. P. CMORPH: A method that produces global precipitation estimates from passive microwave and infrared data at high spatial and temporal resolution. Journal of Hydrometeorology, v. 5, p.487-503, 2004. https://doi.org/10.1175/1525-7541(2004)005<0487:CAMTPG>2.0.CO;2

MAGALHÃES, M.; LIMA, A.C. Noções de probabilidade e estatística. 4. ed. São Paulo: Editora da Universidade de São Paulo, 2002.

MAIOR, M. M. S.; CÂNDIDO, G. A. Avaliação das metodologias brasileiras de vulnerabilidade socioambiental como decorrência da problemática urbana no Brasil. Caderno Metropolitano, v. 16, n. 31, p. 241-264, 2014. 
MARENGO, J. A. On the hydrological cycle of the Amazon Basin: a historical review and current State-of-the-art. Revista Brasileira de Meteorologia, v. 21, n. 3a, p. 01-19, 2006.

MARENGO, J. A.; NOBRE, C. A.; TOMASELlA, J.; OYAMA, M. D.; SAMPAIO DE OLIVEIRA, G.; DE OLIVEIRA, R. et al. The drought of Amazonia in 2005. Journal of Climate, v. 21, p. 495-516, 2008.

MATOS, A. P.; COHEN, J. C. P. Circulação de brisa e a banda de precipitação na margem leste da baia do Marajó. Ciência e Natura, V. 38, p. 21-27, 2016. http://dx.doi.org/10.5902/2179460X19814

MENDONÇA, F. Clima, tropicalidade e saúde: uma perspectiva a partir da intensificação do aquecimento global. Revista Brasileira de Climatologia, v. 1, n. 1, p. 110-112, 2005. http://dx.doi.org/10.5380/abclima.v1i1.25231

MINGOTI, S. A. Análise de dados através de métodos de estatística multivariada: uma abordagem aplicada. Belo Horizonte: Editora UFMG, 2005.

MORAES, B. C. Variação espacial e temporal da precipitação no estado do Pará. Acta Amazônica, v. 35, p. 207-214, 2005.

O'BRIEN, K. L.; ERIKSEN, S.; NYGAARD, L. P.; SCHJOLDEN, A. Why different interpretations of vulnerability matter in climate change discourses. Climate Policy, v. 7, n. 1, p. 73-88, 2013. https://doi.org/10.3763/cpol.2007.0706

PARÁ. Secretária de Estadual de Saúde Pública. Dados epidemiológicos. Belém, 2016.

PEREIRA, F. da S.; VIEIRA, I. C. G. Expansão urbana da região metropolitana de Belém sob a ótica de um sistema de índices de sustentabilidade. Revista Ambiente \& Água, v. 11, n. 3, p. 731-744, 2016. http://dx.doi.org/10.4136/ambi.agua.1878

SODRÉ, G. R. C.; RODRIGUES, L. L. M. comparação entre estimativa da precipitação observada pela técnica CMORPH e estações meteorológicas do INMET em diferentes regiões do Brasil. Revista Brasileira de Geografia Física, v. 6, n. 2, p. 301-307, 2013.

TAVARES, J. P. N.; MOTA, M. A. S. da. Condições termodinâmicas de eventos de precipitação extrema em Belém-PA, durante a estação chuvosa. Revista Brasileira de Meteorologia, v. 27, n. 2, p. 207-218, 2012.

TIBÚRCIO, L. H.; CORRÊA, M. D. P. Análise da vulnerabilidade da microrregião de Itajubá por meio do IVG com vistas à mitigação dos impactos causados pelas mudanças climáticas. Ambiente e Sociedade, v. 15, n. 3, p. 123-139, 2012. http://dx.doi.org/10.1590/S1414-753X2012000300008

TRAVASSOS DA ROSA, A. P. A.; VASCONCELOS, P. F. C.; TRAVASSOS DA ROSA, E. S.; RODRIGUES, S. G.; MONDET, B.; CRUZ, A. C. R. et al. Dengue Epidemic in Belém, Pará, Brazil, 1996-97. Emerging Infectious Disease, v. 6, n. 3, 2000. https://dx.doi.org/10.3201\%2Feid0603.000311

TRIOLA, M. F. Introdução à estatística. 10. ed. Rio de Janeiro: LTC, 2008.

WISNER, B. Vulnerability. International Encyclopedia of Human Geography, p. 176- 182, 2009.

ZAIANE, O. R.; OLIVEIRA, S. R. M. On data clustering analysis: scalability, constraints and validation. Edmonton: University of Alberta, 2003. 